TRANSACTIONS OF THE

AMERICAN MATHEMATICAL SOCIETY

Volume 191, 1974

\title{
AN APPROACH TO FIXED-POINT THEOREMS ON UNIFORM SPACES
}

BY

\section{E. TARAFDAR}

ABSTRACT. Diaz and Metcalf [2] have some interesting results on the set of successive approximations of a self mapping which is either a nonexpansion or a contraction on a metric space with respect to the set of fixed points of the mapping. We have extended most of these results to a Hausdorff uniform space. We have also proved a Banach's contraction mapping principle on a complete Hausdorff uniform space and indicated some applications in locally convex linear topological spaces.

Introduction. Let $(X, d)$ be a metric space. Then a mapping $f$ of $X$ into itself is called a contraction on $X$ if there exists a real number $r$ with $0<r<1$ such that $d(f(x), f(y)) \leq r d(x, y)$ for all points $x$ and $y$ in $X$. Banach's contraction mapping principle states that if $(X, d)$ is a complete metric space and $f$ is a contraction on $X$, then $f$ has a unique fixed point $a \in X$ such that $f^{n}(x) \rightarrow a$ for each $x \in X$. This principle is well known for its wide scope of applications in analysis. It would, therefore, be of some interest to extend this principle in complete Hausdorff uniform spaces which are generalizations of complete metric spaces.

Let $(X, b)$ be a uniform space, $b$ being the uniformity, i.e., the family of entourages. Taylor [1] has introduced the following definitions:

Let $\Re$ be a base for $b$. If $f$ maps $X$ into itself; then

(a) $f$ is said to be $B$-nonexpansion on $X$ if $(x, y) \in H$ implies $(f(x), f(y)) \in H$ for each $H \in \mathfrak{B}$.

(b) $f$ is said to be a $B$-contraction on $X$ if, for each $H \in \mathscr{B}$, there is a $K \in \mathscr{B}$ such that $(x, y) \in H \circ K$ implies $(f(x), f(y)) \in H$.

(c) $f$ is said to be asymptotically regular if for each $x \in X$ and entourage $H \in b$ there is a positive integer $n_{0}$ such that $\left(f^{n}(x), f^{n+1}(x)\right) \in H$ for $n \geq n_{0}$.

The following result is obtained in [1, Lemma 1.5].

Let $(X, b)$ be a complete well-chained Hausdorff uniform space and $B$ a base for $b$. If $f$ is a $B$-contraction on $X$, then $f$ has a unique fixed point $a \in X$ such that $f^{n}(x) \rightarrow a$ for each $x \in X$. (For definition of well-chained uniform space see [1, p. 166].)

Received by the editors May 8, 1973 and, in revised form, July 23, 1973.

AMS (MOS) subject classifications (1970). Primary 47H10; Secondary 54E15; 46 A05.

Key words and phrases. Uniform space, locally convex linear topological space, family of pseudometrics, family of seminorms, nonexpansion, contraction, asymptotically regular maps, contraction mapping principle, starshaped subset. 
This result is not an exact analogue of Banach's contraction mapping principle in the sense that an additional condition of the space being well chained is imposed on $X$.

In the first section of this paper by giving a suitable definition of contraction mapping on a uniform space (which will reduce to the well-known definition of contraction mapping stated in the beginning when the uniform space is a metric space) we have obtained an exact analogue of Banach's contraction mapping principle on a complete Hausdorff uniform space.

In [2] Diaz and Metcalf have obtained a series of results on the cluster set of successive approximations in a metric space by using mainly the nonexpansion and contraction of a mapping with respect to the set of fixed points of the mapping. The main source of this work of Diaz and Metcalf is a paper of Tricomi [3] which is concerned with iteration of a real function. In $\$ 2$ of our paper we have shown that most of the results of [2] can be extended to a uniform space.

In the concluding section we have indicated some applications of our results in locally convex linear topological spaces. Finally we should point out that the theory of nonexpansive mappings is growing very rapidly and a good account of the existing literature can be obtained in [4].

1. Let $(X, b)$ be a uniform space, $b$ being the uniformity. The uniform topology induced by $b$ will be denoted by $\tau_{b}$. A family $\left[\rho_{a}: a \in I\right]$ of pseudometrics on $X$ is called an associated family for the uniformity $b$ on $X$ if the family $[H(\alpha, \epsilon): \alpha \epsilon I, \epsilon>0]$, where $H(\alpha, \epsilon)=\left[(x, y): \rho_{\alpha}(x, y)<\epsilon\right]$, is a subbase for $b$. (For definition of subbase and base for $b$ see Kelly [5].) A family [ $\left.\rho_{a}: a \in I\right]$ of pseudometrics on $X$ is called an augmented associated family for $b$ if $\left[\rho_{a}: a \in I\right]$ is an associated family for $b$ and has the additional property that, given $\alpha, \beta \in I$, there is a $\nu \in I$ such that $\rho_{\nu}(x, y) \geq \max \left(\rho_{\alpha}(x, y), \rho_{\beta}(x, y)\right)$ for all $(x, y) \epsilon$ $X \times X$. An associated family and an augmented associated family for $b$ will be respectively denoted by $A(b)$ and $A^{*}(b)$.

It is well known that if $(X, b)$ is a uniform space and $\left[\rho_{a}: \alpha \in I\right]=A^{*}(b)$, then the family $[H(\alpha, \epsilon): \alpha \in I, \epsilon>0]$ is a base for $b$ (see Thron $[6, \mathrm{p} .179]$, or [5, pp. 188-189]). It is also well known that for each uniformity $b$ on $X$, there exists a family $\left[\rho_{a}: \alpha \in I\right]$ of pseudometrics on $X$ such that $\left[\rho_{a}: a \in I\right]=A^{*}(b)$, and that every family $\left[\rho_{a}: \alpha \in J\right]$ of pseudometrics on $X$ determines a unique uniformity $b$ on $X$ such that $A(b)=\left[\rho_{a}: a \in J\right]$ and $A(b)$ can be enlarged to $A^{*}(b)$ by adjoining to $A(b)$ all the pseudometrics of the form $\max \left[\rho_{a_{k}}: k=1,2, \cdots, n\right]$, where $\left[a_{1}, a_{2}, \cdots, a_{n}\right]$ is an arbitrary finite subset of the index set $J$ (for details see $[6$, p. 177]).

We now make the following definitions. Let $(X, b)$ be a uniform space and let $\left[\rho_{a}: a \in I\right]=A^{*}(b)$. If $f$ maps $X$ into itself, then 
(i) $f$ will be said to be $A^{*}(b)$-nonexpansion on $X$, or simply nonexpansion on $X$ if, for each $a \in I, \rho_{\alpha}(f(x), f(y)) \leq \rho_{\alpha}(x, y)$ for all $(x, y) \in X \times X$.

(ii) $f$ will be said to be $A^{*}(b)$-contraction on $X$, or simply contraction on $X$ if for each $\alpha \in I$, there exists a real number $r(\alpha)$ with $0<r(\alpha)<1$ such that for all $(x, y) \in X \times X$ we have $\rho_{a}(f(x), f(y)) \leq r(a) \rho_{a}(x, y)$ (we note that the above inequality implies $\rho_{a}(f(x), f(y))=0$ if $\left.\rho_{a}(x, y)=0\right)$.

(iii) $f$ will be said to be $A^{*}(b)$-asymptotically regular on $X$, or simply asymptotically regular on $X$ if for each $x \in X$ and $\alpha \in I$,

$$
\lim _{n \rightarrow \infty} \rho_{a}\left(f^{n}(x), f^{n+1}(x)\right)=0
$$

Remark 1. If $f$ is $A^{*}(b)$-nonexpansion, -contraction, or -asymptotically regular on $X$, then it is trivial to see that $f$ is also respectively $A(b)$-nonexpansion, -contraction, or asymptotically regular on $X$. The converse of this is also true, i.e., if $f$ is $A(b)$-nonexpansion, -contraction, or -asymptotically regular on $X$, then $f$ is respectively $A^{*}(b)$-nonexpansion, -contraction, or -asymptotically regular on $X$. We prove it for the case of contraction. The case of nonexpansion and asymptotically regularity follows similarly.

Let $f$ be $A(b)$-contraction. Let $A(b)=\left[\rho_{a}: a \in J\right]$. Let $\rho \in A^{*}(b)$ be arbitrary. If $\rho \in A(b)$, then $\rho=\rho_{a}$ for some $\alpha \in \mathrm{J}$. Hence there will exist a real number $r(a)$ with $0<r(a)<1$ satisfying the condition of definition (ii) as $f$ is $A(b)$ contraction. If $\rho \notin A(b)$, then $\rho=\max \left[\rho_{a_{k}}: k=1,2, \cdots, n\right]$ for some finite subset $\left[a_{1}, a_{2}, \cdots, a_{n}\right]$ of $J$. Let $r=\max \left[r\left(a_{k}\right): k=1,2, \cdots, n\right]$, where $r\left(a_{k}\right)$ 's are obtained from the definition of $A(b)$-contraction of $f$. We will assert that $r$ which clearly satisfies the relation $0<r<1$ is the required number for $\rho$. Let $(x, y) \in X \times X$. Then $\rho(x, y)=\rho_{a_{m}}(x, y)$ for some $m=1,2, \cdots, n$ and $\rho(f(x), f(y))=\rho_{a_{j}}(f(x), f(y))$ for some $j=1,2, \cdots, n$.

Now noting that $f$ is $A(b)$-contraction, and $\rho_{a_{j}} \in A(b)$ and that $\rho_{a_{j}}(x, y) \leq$ $\rho_{a_{m}}(x, y)$, we have

$$
\begin{aligned}
\rho(f(x), f(y)) & =\rho_{a_{j}}(f(x), f(y)) \leq r\left(a_{j}\right) \rho_{a_{j}}(x, y) \leq r\left(a_{j}\right) \rho_{a_{m}}(x, y) \\
& =r\left(a_{j}\right) \rho(x, y) \leq r \rho(x, y) \quad \text { as } r\left(a_{j}\right) \leq r
\end{aligned}
$$

Clearly $r$ depends on $\alpha_{1}, a_{2}, \cdots, \alpha_{n}$ and hence on $\beta$ for which $\rho=\rho_{\beta} \epsilon$ $A^{*}(b)=\left[\rho_{a}: a \in I\right]$.

Thus we see that it does not matter whether we use $A(b)$ or $A^{*}(b)$ in the above definitions.

Remark 2. It is easy to see that the definition (c) of Taylor for asymptotic regularity stated in the introduction is equivalent to our definition (iii) above. 
Also, if we take $B=[H(\alpha, \epsilon): \alpha \in I, \epsilon>0]$, where $\left[\rho_{a}: \alpha \in I\right]=A^{*}(b)$, then we see that definition (a) for $B$-nonexpansion coincides with definition (i) above for nonexpansion. However, definition (b) for contraction is not, in general, equivalent to the above definition (ii) for contraction. One can see this by comparing our Theorem 1.1 and the first lemma and the discussion following this lemma in [1].

Remark 3. If $(X, b)$ is replaced by a metric space $(X, d)$, then the above definitions (i), (ii) and (iii) reduce respectively to the well known definitions for nonexpansion, contraction and asymptotic regularity on the metric space $(X, d)$.

Theorem 1.1 (Banach's Contraction Mapping Principle). Let $(X, b)$ be a Hausdorff complete uniform space and let $\left[\rho_{a}: a \in I\right]=A^{*}(b)$. Let $f$ be a contraction on $X$.

Then $f$ has a unique fixed point $a \in X$ such that $f^{n}(x) \rightarrow a$ in $r_{b}$-topology for eacb $x \in X$.

Proof. Let $x_{0} \in X$. Let $x_{n}=f\left(x_{n-1}\right)=f^{n}\left(x_{0}\right), n=1,2, \ldots$ Let $a \in I$ be arbitrary. If $m$ and $n$ are positive integers with $m<n$, then

$$
\begin{aligned}
\rho_{a}\left(x_{m}, x_{n}\right) & =\rho_{a}\left(f^{m}\left(x_{0}\right), f^{n}\left(x_{0}\right)\right)=\rho_{a}\left(f^{m}\left(x_{0}\right), f^{m} f^{n-m}\left(x_{0}\right)\right) \\
& \leq\{r(a)\}^{m} \rho_{a}\left(x_{0}, f^{n-m}\left(x_{0}\right)\right)=\{r(a)\}^{m} \rho_{a}\left(x_{0}, x_{n-m}\right) \\
& \leq\{r(a)\}^{m}\left[\rho_{a}\left(x_{0}, x_{1}\right)+\rho_{a}\left(x_{1}, x_{2}\right)+\cdots+\rho_{a}\left(x_{n-m-1}, x_{n-m}\right)\right] \\
& \leq\{r(a)\}^{m} \rho_{a}\left(x_{0}, x_{1}\right)\left[1+r(a)+\cdots+\{r(a)\}^{n-m-1}\right] \\
& <\{r(a)\}^{m} \rho_{\alpha}\left(x_{0}, x_{1}\right) /(1-r(a)) \rightarrow 0 \text { as } m, n \rightarrow \infty .
\end{aligned}
$$

Hence $\left\{x_{n}\right\}_{n=1}^{\infty}$ is a $\rho_{\alpha}$-Cauchy sequence (i.e. a Cauchy sequence in $\rho_{\alpha}$-topology). Since $a \in I$ is arbitrary, $\left\{x_{n}\right\}_{n=1}^{\infty}$ is a $\rho_{a}$-Cauchy sequence for each $\alpha \in I$. Let $S_{p}=\left\{x_{n}: n \geq p\right\}$ for all positive integers $p$ and let $\mathbb{B}$ be the filter basis $\left\{S_{p}: p=1,2, \ldots\right\}$. Then, since $\left\{x_{n}\right\}_{n=1}^{\infty}$ is a $\rho_{a}$-Cauchy sequence for each $a \in I$, it is easy to see that the filter basis $\mathcal{B}$ is Cauchy in the uniform space $(X, b)$. To see this we first note that the family $[H(\alpha, \epsilon): a \epsilon I, \epsilon>0]$ is a base for $b$ as $A^{*}(b)=\left[\rho_{a}: a \in I\right]$. Now let $H \in b$ be an arbitrary entourage. Then there exist a $\nu \in I$ and $\epsilon>0$ such that $H(\nu, \epsilon) \subset H$. Now since $\left\{x_{n}\right\}_{n=1}^{\infty}$ is a $\rho_{\nu}$-Cauchy sequence in $X$, there exists a positive integer $p$ such that $\rho_{\nu}\left(x_{m}, x_{n}\right)<\epsilon$ for $m \geq p, n \geq p$. This implies that $S_{p} \times S_{p} \subset H(\nu, \epsilon)$. Thus given any $H \in b$, we can find a $S_{p} \in \mathfrak{B}$ such that $S_{p} \times S_{p} \subset H$. Hence $B$ is a Cauchy filter in $(X, b)$. Since $(X, b)$ is complete and Hausdorff, the Cauchy filter $B=\left\{S_{p}\right\}$ converges to a unique point $a \in X$ in the $\tau_{b}$-topology. Thus $\tau_{b}$-lim $S_{p}=a$. Now since $f$ is $\rho_{a}$-continuous for each $a \in I$, it follows that $f$ is $r_{b}$-continuous. Hence $f(a)=f\left(r_{b}-\lim S_{p}\right)=$ $\tau_{b}-\lim f\left(S_{p}\right)=\tau_{b}-\lim S_{p+1}=a$. Thus $a$ is a fixed point of $f$

We now complete the proof of our theorem by showing that $a$ is the only fixed point of $f$. We assume that $f$ has another fixed point $b$ such that $a \neq b$ and deduce an absurdity from this assumption. Since $(X, b)$ is a Hausdorff space and $a \neq b$, 
there is an index $\beta \in I$ such that $\rho_{\beta}(a, b) \neq 0$. Since $f$ is a contraction on $X$, $\rho_{\beta}(a, b)=\rho_{\beta}(f(a), f(b)) \leq r(\beta) \rho_{\beta}(a, b)$ which is absurd as $0<r(\beta)<1$ and $\rho_{\beta}(a, b) \neq 0$. This together with the fact that $\tau_{b}-\lim S_{p}=a$ implies $\tau_{b}-\lim x_{n}=a$ completes the proof.

2. In this section we extend some of the results of Diaz and Metcalf [2] to Hausdorff uniform spaces. To start with we write few facts in the form of lemmas.

Lemma 2.1. Let $(X, b)$ be a uniform space and let $\left[\rho_{a}: a \in I\right]=A^{*}(b)$. If $X$ is $r_{b}$-compact, then $X$ is $\rho_{a}$-compact for eacb $a \in I$. (A topological space $(X, r)$ is r-compact if every r-open covering of $X$ bas a finite subcovering.)

Lemma 2.2. Let $(X, b)$ be a uniform space and let $\left[\rho_{a}: a \in I\right]=A^{*}(b)$. Let $\left\{x_{n}\right\}_{n \in J}$ be a net in $X$.

(a) If $\left\{x_{n}\right\}_{n} \in_{J}$ is $\tau_{b}$-convergent and converges to $x$, then for each $a \in I$, it is $\rho_{a}$-convergent and converges to $x$. Conversely, if $\left\{x_{n}\right\}_{n \in J}$ is $\rho_{a}$-convergent and converges to $x$ for eacb $a \in I$, then it is $\tau_{b}$-convergent and converges to $x$.

(b) If $y$ is a $r_{b}$-cluster point of the net $\left\{x_{n}\right\}_{n \in J}$ then $y$ is a $\rho_{a}$-cluster point of the net $\left\{x_{n}\right\}_{n \in J}$ for each $a \in I$.

The proofs of the above two lemmas are trivial and hence omitted.

Lemma 2.3. Let $(X, b)$ be a Hausdorff uniform space and let $\left[\rho_{a}: \alpha \in I\right]=$ $A^{*}(b)$. If $A$ and $B$ are a disjoint pair of $\tau_{b}$-compact subsets of $X$, then there exists at least one $\beta \in I$ sucb that $\rho_{\beta}(A, B)>0$.

Proof. Since $X$ is $\tau_{b}$-Hausdorff, it follows that $A$ and $B$ are disjoint $\tau_{b}{ }^{-}$ closed and $\tau_{b}$-compact subsets of $X$. Hence we can find a symmetric entourage $W \in b$ such $W(A) \cap W(B)=\varnothing$ (e.g. see [7, Theorem 5, p. 117]) where for any subset $C$ of $X, W(C)=\{y \in X:(x, y) \in W, x \in C\}$; i.e., $W(C)=\bigcup_{x \in C} W(x)$ where $W(x)$ $=\{y \in x:(x, y) \in W\}$.

With this symmetric entourage $W$ we can construct a sequence $\left\{W_{n}\right\}$ of symmetric entourages such that $W_{n} \circ W_{n} \circ W_{n} \subset W_{n-1}, W_{1}=W \cap W^{-1}=W$ and $W_{0}=$ $X$ and then we can show that there exists a pseudometric $\rho \in A^{*}(b)$ such that for each positive integer $n, W_{n} \subset\left[(x, y): \rho(x, y) \leq 2^{-n}\right] \subset W_{n-1}$ (for details see Thron [6, pp. 178-179]). We first assert that for no pair $(x, y)$ of points with $x \in A$, $y \in B$ we have $\rho(x, y)=0$. We suppose contrary to what we wish to prove that for some pair $(p, q)$ of points with $p \in A$ and $q \in B$, we have $\rho(p, q)=0$. Then since $\rho(p, q)=0,(p, q) \in W_{n}$ for each $n$ and hence $(p, q) \in W_{1}=W$ in particular. Then clearly $p \in W(A)$ and $p \in W(B)$. This contradicts that $W(A) \cap W(B)=\varnothing$. Thus we have proved our assertion. Next we prove that $\rho(A, B)>0$. Since $\rho \in A^{*}(b)$ and $A$ and $B$ are $\tau_{b}$-compact, we have by Lemma 2.1 that $A$ and $B$ are both $p$-compact. 
Hence, if $\rho(A, B)=0$, then there would exist a pair $(x, y)$ of points with $x \in A$ and $y \in B$ such that $\rho(x, y)=0$ which would contradict our established assertion that $\rho(x, y) \neq 0$ for each pair $(x, y)$ of points with $x \in A$ and $y \in B$. Hence $\rho(A, B)>0$. As $\rho \in A^{*}(b), \rho=\rho_{\beta}$ for some $\beta \in I$. Thus we have proved the lemma.

Notation. Let $(X, b)$ be a uniform space and let $f: X \rightarrow X$ be a mapping of $X$ into $X$. Then $F(f)$ will be the set of fixed points of $f$, i.e., $F(f)=\{x \in X$ : $f(x)=x\}$. For any $x \in X, L(x)$ will denote the set of all cluster points of the net (sequence) of iterates $\left\{f^{n}(x)\right\}_{n=1}^{\infty}$, i.e. $L(x)$ is the set of all $y \in X$ such that $\left\{f^{n_{i}}(x)\right\}_{i \in J} \rightarrow y$ in $r_{b}$ topology for some subnet $\left\{f^{n}(x)\right\}_{i \in J}$ of the net $\left\{f^{n}(x)\right\}_{n=1}^{\infty}$.

The following is an extension of Theorem 6 in [2] to the uniform space.

Theorem 2.1 Let $(X, b)$ be a nonempty Hausdorff uniform space and let $\left[\rho_{a}: \alpha \in I\right]=A^{*}(b)$. Let $f: X \rightarrow X \tau_{b}$-continuous. Also let

(a) $f(X)$ be $r_{b}$-compact; and

(b) $f$ be asymptotically regular on $X$.

Then for each $x \in X$, the $\tau_{b}$-cluster set $L(x)$ is a nonempty $\tau_{b}$-closed and $r_{b}$-connected subset of $F(f)$. In case $L(x)$ is just one point, then $r_{b}-\lim f^{m}(x)$ exists and belongs to $F(f)$. In case $L(x)$ contains more than one point, then it is contained in the $\tau_{b}$-boundary of $F(f)$. [The $\tau_{b}$-boundary of a subset $K$ of $X=$ $r_{b}$-closure of $K-\tau_{b}$-Int $K$ where Int $K$ stands for the interior of $\left.K.\right]$

Proof. The sequence $\left\{f^{n}(x)\right\}_{n=1}^{\infty}$ being also a net in the compact set $f(X)$ has a cluster point. Hence $L(x)$ is nonempty. We prove the rest of the theorem in few steps.

(i) $L(x)$ is a subset of $F(f)$. Let $y \in L(x)$. Then there is a subnet $\left\{f^{n} j(x)\right\}_{j \in J}$ of the net $\left\{f^{n}(x)\right\}_{n=1}^{\infty}$ such that $f^{n} j(x) \rightarrow y$ in $\tau_{b}$-topology. Also since $f$ is $\tau_{b}{ }^{-}$ continuous, the net $f^{n_{j}+1}(x) \rightarrow f(y)$ in $\tau_{b}$-topology. Hence by Lemma 2.2, for each $\alpha \in I$, the net $f^{n j(x)} \rightarrow y$ and the net $f^{n_{j+1}}(x) \rightarrow f(y)$ in the $\rho_{a}$ topology of $X$. Let $a \in I$ be arbitrary. Then for each $j \in J$, we have

$$
\rho_{a}(f(y), y) \leq \rho_{a}\left(f(y), f^{n^{+1}}(x)\right)+\rho_{a}\left(f^{n^{+1}}(x), f^{n} j(x)\right)+\rho_{a}\left(f^{n} j(x), y\right) .
$$

Let $\epsilon>0$ be arbitrarily chosen. Then since in $\rho_{a}$-topology of $X, f^{n_{j}^{+1}}(x)$ $\rightarrow f(y)$ and $f^{n} j(x) \rightarrow y$ and since by asymptotic regularity we have $\rho_{a}\left(f^{n_{j}+1}(x), f^{\left.n_{j}(x)\right)} \rightarrow 0\right.$, we can find a $p \in J$ such that for all $n_{j} \geq n_{p}$ we have simultaneously

(2) $\rho_{a}\left(f^{n} j^{+1}(x), f(y)\right)<\epsilon / 3, \quad \rho_{a}\left(f^{n^{+1}}(x), f^{n} j(x)\right)<\epsilon / 3$, and $\rho_{a}\left(f^{n} j(x), y\right)<\epsilon / 3$.

Now from (1) and (2) we have $\rho_{a}(f(y), y)<\epsilon$. Since $\epsilon$ is arbitrary, $\rho_{a}(f(y), y)=0$. Again since $a$ is arbitrary, $\rho_{a}(f(y), y)=0$ for each $a \in I$. Finally since $X$ is Hausdorff, it follows that $f(y)=y$. (The uniform space $X$ is Hausdorff iff, given 
two distinct points $x$ and $y$, there is a $\beta \in I$ such that $\rho_{\beta}(x, y) \neq 0$.)

(ii) $L(x)$ is a $r_{b}$-closed subset of $F(f)$. It is well known that the cluster set of any net is always closed. Thus $L(x)$ is a $r_{b}$-closed subset of $F(f)$ as we have proved in (i) that $L(x) \subset F(f)$.

(iii) We now prove that $L(x)$ is a $r_{b}$-connected subset of $F(f)$. Although our proof is similar to the proof of the corresponding part of Theorem 6 in [2], our Lemma 2.3 will play the crucial role. If $L(x)$ consists of a single point, then there is nothing to prove. So we may suppose that $L(x)$ consists of more than one point. We assume that $L(x)$ is not a $\tau_{b}$-connected subset of $F(f)$ and deduce a contradiction from this assumption. Since $L(x)$ is not a $r_{b}$-connected subset of $F(f), L(x)=S_{1} \cup S_{2}$ where $S_{1}$ and $S_{2}$ are both nonempty and $\tau_{b}$-closed and $S_{1}$ $\cap S_{2}=\varnothing$. Also since $f(X)$ is compact, it follows that $S_{1}$ and $S_{2}$ are both $r_{b}{ }^{-}$ compact. Thus $S_{1}$ and $S_{2}$ are disjoint $\tau_{b}$-closed and $\tau_{b}$-compact subsets of $X$.

Now for each $a \in I$, let

$$
S_{1}^{a}=\left\{y \in F(f): \rho_{a}\left(y, S_{1}\right) \leq 1 / 4 \rho_{a}\left(S_{1}, S_{2}\right)\right.
$$

and

$$
S_{2}^{\alpha}=\left\{y \in F(f): \rho_{a}\left(y, S_{2}\right) \leq 1 / 4 \rho_{a}\left(S_{1}, S_{2}\right) .\right.
$$

$F(f)$ being a $\tau_{b}$-closed subset of $\tau_{b}$-compact set $f(X)$ is $\tau_{b}$-compact. Hence $F(f)$ is by Lemma $2.1 \rho_{a}$-compact for each $\alpha \in I$. Hence $S_{1}^{a}$ and $S_{2}^{a}$ being $\rho_{a}$-closed in $F(f)$ are both $\rho_{a}$-compact subsets of $F(f)$ for each $\alpha \in I$. .

We first prove that $\lim _{m \rightarrow \infty} \rho_{a}\left(f^{m}(x), s_{1}^{a} \cup s_{2}^{a}\right)=0$ for each $a \in l$. We suppose that this is not true for some $\beta \in I$ and obtain a contradiction. Then there would exist $\epsilon>0$ and a subsequence $\left\{f^{n}(x)\right\}_{i=1}^{\infty}$ of the sequence $\left\{f^{m}(x)\right\}_{m=1}^{\infty}$ such that

$$
\rho_{\beta}\left(f^{n} i(x), s_{1}^{\beta} \cup s_{2}^{\beta}\right) \geq \epsilon>0 \quad \text { for each } i=1,2, \ldots .
$$

Now the subsequence $\left\{f^{n} i(x)\right\}_{i=1}^{\infty}$ being a net in the $r_{b}$-compact set $f(X)$ has a cluster point, say, $z$. Then obviously $z \in L(x)$ and $z$ is a $\rho_{\beta}$-cluster point of the sequence $\left\{f^{n_{i}}(x)\right\}_{i=1}^{\infty}$ by Lemma 2.2. Hence there is a subsequence $\left\{f^{\left.n_{p_{j}}(x)\right\}_{j=1}^{\infty}}\right.$ of the sequence $\left\{f^{n_{i}}(x)\right\}_{i=1}^{\infty}$ such that $\lim _{j \rightarrow \infty} \rho_{\beta}\left(f^{n_{p_{j}}}(x), z\right)=0$ because $\rho_{\beta}$-topology of $X$ satisfies the first axiom of countability. Now since $z \in L(x)=S_{1} \cup S_{2} \subset S_{1}^{\beta} \cup S_{2}^{\beta}$, we have

Hence

$$
\rho_{\beta}\left(f^{n}{ }^{p_{j}}(x), s_{1}^{\beta} \cup s_{2}^{\beta}\right) \leq \rho_{\beta}\left(f^{n_{p}}(x), z\right), \quad j=1,2, \ldots
$$

which contradicts (3).

$$
\lim _{j \rightarrow \infty} \rho_{\beta}\left(f^{n}{ }_{j}(x), s_{1}^{\beta} \cup s_{2}^{\beta}\right)=0
$$


Thus we have proved that

$$
\lim _{m \rightarrow \infty} \rho_{a}\left(f^{m}(x), s_{1}^{a} \cup S_{2}^{a}\right)=0 \text { for each } a \in I .
$$

Since $S_{1}$ and $S_{2}$ are disjoint, $\tau_{b}$-closed and $\tau_{b}$-compact subsets of $X$, there exists by Lemma 2.3 at least one $\nu \in I$ such that $\rho_{\nu}\left(s_{1}, s_{2}\right)>0$. We now prove that $S_{1}^{\nu}$ and $S_{2}^{\nu}$ are disjoint. If $p \in S_{1}^{\nu} \cap S_{2}^{\nu}$ then, since $S_{1}$ and $S_{2}$ are both $\rho_{\nu}-$ compact subsets (by Lemma 2.1) of $F(f)$, there would exist points $a \in S_{1}$ and $b \in S_{2}$ such that $\rho_{\nu}\left(p, S_{1}\right)=\rho_{\nu}(a, p)$ and $\rho_{\nu}\left(p, S_{2}\right)=\rho_{\nu}(p, b)$.

$$
\text { Hence } 0<\rho_{\nu}\left(s_{1}, s_{2}\right) \leq \rho_{\nu}(a, b) \leq \rho_{\nu}(a, p)+\rho_{\nu}(p, b)=\rho_{\nu}\left(p, s_{1}\right)+\rho_{\nu}\left(p, s_{2}\right)
$$

$\leq 1 / 2 \rho_{\nu}\left(S_{1}, S_{2}\right)$ which is absurd. Thus $S_{1}^{\nu}$ and $S_{2}^{\nu}$ are disjoint. Also we have noted earlier that both $S_{1}^{\nu}$ and $S_{2}^{\nu}$ are $\rho_{\nu}$-closed and $\rho_{\nu}$-compact subsets of $F(f)$. Hence $\rho_{\nu}\left(S_{1}^{\nu}, s_{2}^{\nu}\right)>0$. In summary we have

$\rho_{\nu}\left(s_{1}^{\nu}, s_{2}^{\nu}\right)>0 ; \quad \lim _{m \rightarrow \infty} \rho_{\nu}\left(f^{m}(x), f^{m+1}(x)\right)=0 \quad$ and $\quad \lim _{m \rightarrow \infty} \rho_{\nu}\left(f^{m}(x), s_{1}^{\nu} \cup s_{2}^{\nu}\right)=0$.

In view of the last two limits we can find a positive integer $M$ such that, for all $m \geq M$,

$$
\rho_{\nu}\left(f^{m}(x), f^{m+1}(x)\right)<\rho_{\nu}\left(s_{1}^{\nu}, s_{2}^{\nu}\right) / 3 \text { and } \rho_{\nu}\left(f^{m}(x), s_{1}^{\nu} \cup s_{2}^{\nu}\right)<\rho_{\nu}\left(s_{1}^{\nu}, s_{2}^{\nu}\right) / 3 \text {. }
$$

The rest of the proof is similar to that in [2] and we repeat this for the sake of clarity and completeness. It follows from the last inequality that for any $m \geq M$ we have either

$$
\rho_{\nu}\left(f^{m}(x), s_{1}^{\nu}\right)<\rho_{\nu}\left(s_{1}^{\nu}, s_{2}^{\nu}\right) / 3
$$

or,

$$
\rho_{\nu}\left(f^{m}(x), s_{2}^{\nu}\right)<\rho_{\nu}\left(s_{1}^{\nu}, s_{2}^{\nu}\right) / 3
$$

The set of integers $m \geq M$ satisfying (4) is not empty as $S_{1}$ is not empty and the set of integers $m \leq M$ satisfying $(5)$ is not empty as $S_{2}$ is not empty. Hence we can find a positive integer $n \geq M$ such that we have both

$$
\rho_{\nu}\left(f^{n}(x), s_{1}^{\nu}\right)<\rho_{\nu}\left(s_{1}^{\nu}, s_{2}^{\nu}\right) / 3 \text { and } \rho_{\nu}\left(f^{n+1}(x), s_{2}^{\nu}\right)<\rho_{\nu}\left(s_{1}^{\nu}, s_{2}^{\nu}\right) / 3 \text {. }
$$

[For any $m_{1} \geq M$ such that $\rho_{\nu}\left(f^{m 1}(x), s_{1}^{\nu}\right)<\rho_{\nu}\left(s_{1}^{\nu}, s_{2}^{\nu}\right) / 3$ there always exists a positive integer $m_{2}>m_{1}$ such that $\rho_{\nu}\left(f^{m_{2}}(x), s_{2}^{\nu}\right)<\rho_{\nu}\left(s_{1}^{\nu}, s_{2}^{\nu}\right) / 3$. $n$ can be chosen to be one less than smallest such $m_{2}$.]

By using $\rho_{\nu}$-compactness of $S_{1}^{\nu}$ and $S_{2}^{\nu}$ we have

$$
\rho_{\nu}\left(s_{1}^{\nu}, s_{2}^{\nu}\right) \leq \rho_{\nu}\left(f^{n}(x), s_{1}^{\nu}\right)+\rho_{\nu}\left(f^{n}(x), f^{n+1}(x)\right)+\rho_{\nu}\left(f^{n+1}(x), s_{2}^{\nu}\right) \text {. }
$$

But then by what we have proved above

$$
\rho_{\nu}\left(s_{1}^{\nu}, s_{2}^{\nu}\right)<\rho_{\nu}\left(s_{1}^{\nu}, s_{2}^{\nu}\right) / 3+\rho_{\nu}\left(s_{1}^{\nu}, s_{2}^{\nu}\right) / 3+\rho_{\nu}\left(s_{1}^{\nu}, s_{2}^{\nu}\right)=\rho_{\nu}\left(s_{1}^{\nu}, s_{2}^{\nu}\right)
$$


which is impossible. Hence our original assumption that $L(x)$ is not a $r_{b}$-connected subset of $F(f)$ is wrong. Thus we have proved that $L(x)$ is a $r_{b}$-connected subset of $F(f)$.

(iv) If $L(x)$ consists of a single point, then we prove that $\tau_{b}-\lim f^{m}(x)$ exists. Let us denote the only cluster point of $\left\{f^{m}(x)\right\}_{m=1}^{\infty}$ by $p$. We prove that $\tau_{b}-\lim f^{m}(x)$ $=p$. Let us assume that $\tau_{b}-\lim f^{m}(x) \neq p$ and deduce a contradiction from this assumption. Then there is a subnet $\left\{f^{m k}(x)\right\}_{k \in K}$ of the net $\left\{f^{m}(x)\right\}_{m=1}^{\infty}$ such that $\left\{f^{m_{k}}(x)\right\}_{k \in K}$ has no subnet converging to $p$ in the $\tau_{b}$ topology. But since $\left\{f^{m_{k}}(x)\right\}_{k \in K}$ is a net in the $r_{b}$-compact set $f(X)$, it has a subnet converging to a point, say, $q$ in the $r_{b}$-topology, i.e., it has a $\tau_{b}$-cluster point $q$. Clearly $p \neq q$ and $q$ is also a $r_{b}$-cluster point of the net $\left\{f^{m}(x)\right\}_{m=1}^{\infty}$. Hence $L(x)$ consists of at least two distinct points $p$ and $q$. This contradiction proves that $r_{b}-\lim f^{m}(x)$ $=p$.

(v) Finally we prove that if $L(x)$ consists of more than one point, then it is contained in the $\tau_{b}$-boundary of $F(f)$. Since $L(x)$ consists of more than one point, it is clear that $f^{m}(x) \notin F(f)$ for any $m=0,1,2, \cdots$, where $f^{0}(x)=x$. Let $y \in$ $L(x)$ be arbitrary. If $y$ belonged to Int $F(f)$, then it would follow that Int $F(f)$ would contain $f^{k}(x)$ for some positive integer $k$ which would contradict the assumption that $L(x)$ contains more than one point. Hence $y \in r_{b}$-boundary of $\cdot F(f)$. Thus $L(x) \subset \tau_{b}$-boundary of $F(f)$.

The following theorem is the generalization of some parts of the main result (Theorem 2) of [2] to uniform space.

Theorem 2.2. Let $(X, b)$ be a Hausdorff uniform space and let $\left[\rho_{a}: \alpha \in I\right]=$ $A^{*}(b)$. Let $f: X \rightarrow X$ be $\tau_{b^{-c o n t i n u o u s . ~ A l s o ~ s u p p o s e ~ t h a t ~}}$

(i) $F(f)$ is nonempty and compact;

(ii) for each $x \in X$, with $x \notin F(f)$ we have for each a $\in I, \rho_{a}(f(x), F(f))<$ $\rho_{a}(x, F(f))$ if $\rho_{a}(x, F(f)) \neq 0$ and $\rho_{a}(f(x), F(f))=0$ if $\rho_{a}(x, F(f))=0$.

Then for eacb $x \in X$ the set $L(x)$ is a closed subset of $F(f)$. If $L(x)$ consists of more than one point, then $L(x)$ is contained in the $\tau_{b}$-boundary of $F(f)$.

Proof. We have nothing to prove if $L(x)$ is empty. So we may suppose that $L(x)$ is nonempty. Again, if $x \in F(f)$ or $f^{k}(x) \in F(f)$ for some integer $k \geq 1$, then obviously $\tau_{b}-\lim f^{m}(x)$ exists and belongs to $F(f)$ and thus the eorem is proved in this case. So we prove the theorem in the remaining case, i.e. we assume that $f^{m}(x) \notin F(f)$ for each $m=0,1,2, \ldots$.

(1) First we prove that for each $\alpha \in I, \lim _{m \rightarrow \infty} \rho_{a}\left(f^{m}(x), F(f)\right)$ exists and nonnegative. Let $a$ be arbitrary. Now if $\rho_{a}\left(f^{k}(x), F(f)\right)=0$ for some integer $k=0$, $1,2, \cdots$, then by the second part of condition (ii) of the theorem every element of the sequence $\left\{\rho_{a}\left(f^{m}(x), F(f)\right)\right\}_{m=1}^{\infty}$ starting from the $k$ th element (from the first 
element if $k=0)$ is zero. Consequently $\lim _{m \rightarrow \infty} \rho_{a}\left(f^{m}(x), F(f)\right)=0$. In the remaining case, i.e., when $\rho_{a}\left(f^{m}(x), F(f) \neq 0\right.$ for each $m=0,1,2, \cdots$, the sequence of positive numbers $\left\{\rho_{a}\left(f^{m}(x), F(f)\right\}_{m=1}^{\infty}\right.$ is a decreas ing sequence by virtue of the first part of condition (ii) of the theorem and, therefore, $\lim _{m \rightarrow \infty} \rho_{\alpha}\left(f^{m}(x), F(f)\right)$ exists and is nonnegative.

(2) Next we prove that $L(x)$ is a subset of $F(f)$. Let $y \in L(x)$. It suffices to prove that $y \in F(f)$. We assume that $y \notin F(f)$ and arrive at a contradiction from this assumption. Since $y \in L(x)$, there is a subnet $\left\{f^{n_{j}}(x)\right\}_{j \in J}$ of the net $\left\{f^{m}(x)\right\}_{m=1}^{\infty}$ such that $f^{n} j(x) \rightarrow y$ in the $\tau_{b}$-topology of $X$. Also since $f$ is continous, the net $f^{n_{j}+1}(x) \rightarrow f(y)$ in the $r_{b}$ topology of $X$. Now by our Lemma 2.2, for each $a \in I$, $f^{n_{j}}(x) \rightarrow y$ and $f^{n_{j}+1}(x) \rightarrow f(y)$ in $\rho_{a}$-topology of $X$. The rest of the proof depends on the simple fact that if $\left\{f^{n_{j}}(x)\right\}_{j \in J}$ is a subnet of $\left\{f^{m}(x)\right\}_{m=1}^{\infty}$ then $\left\{f^{n_{j}+1}(x)\right\}_{j \in J}$ is also a subnet of $\left\{f^{m}(x)\right\}_{m=1}^{\infty}$. (1)

Let $a \in I$ be arbitrary. Then noting that in $\rho_{a}$ topology of $X, f^{n} j(x) \rightarrow y$ and $f^{n_{j}+1}(x) \rightarrow f(y)$ and using the continuity of the real valued function $\rho_{a}(x, F(f))$, $x \in X$, we have

$$
\rho_{a}\left(f^{n}(x), F(f)\right) \rightarrow \rho_{a}(y, F(f)) \text { and } \rho_{a}\left(f^{n^{+1}}(x), F(f)\right) \rightarrow \rho_{a}(f(y), F(f)) .
$$

Hence in view of the fact that $\lim _{m \rightarrow \infty} \rho_{a}\left(f^{m}(x), F(f)\right)$ exists and that $\left\{\rho_{a}\left(f^{n_{j}}(x), F(f)\right)\right\}_{j \in J}$ and $\left\{\rho_{a}\left(f^{n_{j}+1}(x), F(f)\right)\right\}_{j \in J}$ are both subnets of the net $\left\{\rho_{a}\left(f^{m}(x), F(f)\right)\right\}_{j \in J}$ of real numbers, we have $\rho_{a}(f(y), F(f))=\rho_{a}(y, F(f))$. Now since by assumption $y \notin F(f)$, the above equality together with condition (ii) of the theorem implies that $\rho_{a}(y, F(f))=0$. Since $\alpha$ is arbitrary, $\rho_{a}(y, F(f))=0$ for each $a \in I$. But this contradicts our Lemma 2.3 as $\{y\}$ and $F(f)$ are dis joint pair of $\tau_{b}$-compact subsets of Hausdorff uniform space $X$. Hence $y \in F(f)$.

(3) That $L(x)$ is closed is well known and the proof that $L(x)$ is in the $\tau_{b}$ boundary of $F(f)$ when it consists of more than one point is exactly the same as given in part $(v)$ of the proof of Theorem 2.1.

The next theorem is an extension of Theorem 3 in [2] to uniform space.

Theorem 2.3. Let $(X, b)$ be a Hausdorff uniform space and let $\left[\rho_{a}: \alpha \in I\right]=$ $A^{*}(b)$. Let $f: X \rightarrow X$ be $r_{b}{ }^{-c o n t i n u o u s . ~ F u r t h e r ~ s u p p o s e ~ t h a t ~}$

(i) $F(f)$ is nonempty;

(ii) for each $x \in X$, with $x \notin F(f)$ and each $p \in F(f)$, we bave for each a $\epsilon$ I, $\rho_{\alpha}(f(x), p)<\rho_{a}(x, p)$ if $\rho_{a}(x, p) \neq 0$, and $\rho_{a}(f(x), p)=0$ if $\rho_{a}(x, p)=0$.

(1) Note that if $n$ is the corresponding function for the first subnet, i.e. $n(j)=n_{j}$ for each $j \in J$, then the function $n^{1}$ defined by $n^{1}(j)=n(j)+1=n_{j}+1$ is the required function for the latter subnet. 
Then for each $x \in X$, either $\left\{f^{m}(x)\right\}_{m=1}^{\infty}$ bas no $\tau_{b}$-convergent subnet, or $r_{b}-\lim f^{m}(x)$ exists and belongs to $F(f)$.

Proof. We have nothing to prove if $L(x)$ is empty. So we may assume that $L(x)$ is nonempty. If $x \in F(f)$, or $f^{k}(x) \in F(f)$ for some integer $k \geq 1$, then obviously $\tau_{b}-\lim f^{m}(x)$ exists and belongs to $F(f)$ and, therefore, the theorem is proved in this case. Hence we assume that $f^{m}(x) \notin F(f)$ for each $m=0,1$, $2, \ldots$ and prove our theorem in the following steps.

(a) In this step we prove that for each $\alpha \in I, \lim _{m \rightarrow \infty} \rho_{a}\left(f^{m}(x), p\right)$ exists and nonnegative, where $p$ is any point belonging to $F(f)$. Such a $p$ exists by condition (i) of the theorem. The proof is similar to the proof of the part 1 of our previous theorem. Let $\alpha \in I$ be arbitrary. Now if $\rho_{a}\left(f^{k}(x), p\right)=0$ for some integer $k=0,1,2, \cdots$, then by the second part of condition (ii) of our theorem each element of the sequence $\left\{\rho_{a}\left(f^{m}(x), p\right)\right\}_{m=1}^{\infty}$ starting from the $k$ th element (from the first element if $k=0)$ is zero and hence $\lim _{m \rightarrow \infty} \rho_{a}\left(f^{m}(x), p\right)=0$ in this case.

If $\rho_{a}\left(f^{m}(x), p\right) \neq 0$ for each $m=0,1,2, \cdots$, then by the first part of condition (ii) of the theorem the sequence of positive numbers $\left\{\rho_{a}\left(f^{m}(x), p\right)\right\}_{m=1}^{\infty}$ is decreasing sequence and, therefore, $\lim _{m \rightarrow \infty} \rho_{\alpha}\left(f^{m}(x), p\right)$ exists and is nonnegative.

(b) We next prove that $L(x)$ is a subset of $F(f)$. Here again the proof is very much similar to the proof of part 2 of the previous theorem. Let $y \in L(x)$. Then there is a subnet $\left\{f^{n_{j}}(x)\right\}_{j \in J}$ of the net $\left\{f^{m}(x)\right\}_{m=1}^{\infty}$ such that $f^{n_{j}}(x) \rightarrow y$ in the $r_{b}$-topology of $X$. Also by the $r_{b}$-conti nuity of $f, f^{n_{j}+1}(x) \rightarrow f(y)$. Hence by our Lemma 2.2, for each $a \in I$ we have that $f^{n_{j}}(x) \rightarrow y$ and $f^{n_{j}+1}(x) \rightarrow f(y)$ in the $\rho_{a}$-topology of $X$. Then by using the above two limits and the continuity of the real valued function $\rho_{a}(x, p), x \in X$, we have

$$
\rho_{a}\left(f^{n} j(x), p\right) \rightarrow \rho_{a}(y, p) \text { and } \rho_{\alpha}\left(f^{n^{+1}}(x), p\right) \rightarrow \rho_{\alpha}(f(y), p) \text {. }
$$

Now, since we have proved in part (a) that $\lim _{m \rightarrow \infty} \rho_{a}\left(f^{m}(x), p\right)$ exists, we have $\rho_{a}(f(y), p)=\rho_{a}(y, p)$ because of the same reason given in part 2 of the previous theorem, i.e. because $\left\{f^{n} j(x)\right\}_{j \in J}$ and $\left\{f^{n_{j}+1}(x)\right\}_{j \in J}$ are both subnets of the net $\left\{f^{m}(x)\right\}_{m=1}^{\infty}$. We now assume that $y \notin F(f)$ and readily deduce a contradiction from this assumption. Since $y \notin F(f)$ and $\rho_{a}(f(y), p)=\rho_{a}(y, p)$, it follows from condition (ii) of our theorem that $\rho_{\alpha}(y, p)=0$. Since $a$ is arbitrary, we have $\rho_{\alpha}(y, p)=$ 0 for each $\alpha \in I$. This implies that $y=p$ as $X$ is $r_{b}$-Hausdorff. This is a contradiction because of the fact that $p \in F(f)$. Thus we have proved that $y \in L(x)$.

(c) We now prove that $L(x)$ contains at most one point. We suppose that $L(x)$ contains two points, $p$ and $q$. By Lemma $2.2, p$ and $q$ are also $\rho_{\alpha}$-cluster points of the net $\left\{f^{m}(x)\right\}_{m=1}^{\infty}$ for each $\alpha \in I$. Let $\alpha$ be arbitrary. Then there are two subsequences $\left\{f^{m i(x)}\right\}_{i=1}^{\infty}$ and $\left\{f^{n} i(x)\right\}_{i=1}^{\infty}$ of the sequence $\left\{f^{m}(x)\right\}_{m=1}^{\infty}$ such 
that $f^{m i}(x) \rightarrow p$ and $f^{n} i(x) \rightarrow q$ in the $\rho_{a}$ topology of $X$ (as the $\rho_{a}$-topology of $X$ satisfies the first axiom of countability) i.e.,

$$
\lim _{i \rightarrow \infty} \rho_{\alpha}\left(f^{m_{i}}(x), p\right)=0 \text { and } \lim _{i \rightarrow \infty} \rho_{\alpha}\left(f^{n_{i}}(x), q\right)=0 \text {. }
$$

We can select a subsequence $\left\{m_{i}^{\prime}\right\}_{i=1}^{\infty}$ of $\left\{m_{i}\right\}_{i=1}^{\infty}$ such that $m_{i}^{\prime}>n_{i}$ for $i=1,2$,

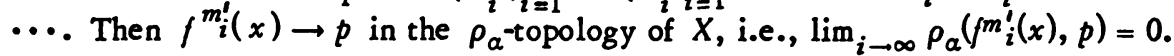
Also we have, $f^{m}(x) \notin F(f)$ for each $m=0,1,2, \ldots$ and $q \in F(f)$ by part (b). We

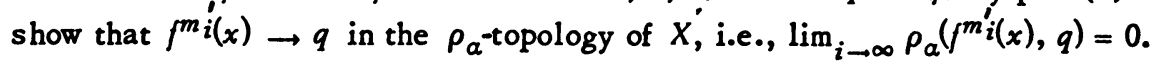

We prove this by considering two cases (A) and (B).

(A) $\rho_{a}\left(f^{m} k(x), q\right)=0$ for some $k=1,2, \ldots$.

Then $\rho_{a}\left(f^{m_{k}+r}(x), q\right)=0$ for each $r=1,2, \ldots$. For, let $m_{k+r}^{\prime}-m_{k}^{\prime}=t$. Then by the second part of condition (ii) of the theorem

$$
0=\rho_{a}\left(f^{m_{k}^{\prime}}(x), q\right)=\rho_{a}\left(f^{m_{k}^{\prime}+1}(x), q\right)=\rho_{a}\left(f^{m_{k}^{\prime}+t}(x), q\right)=\rho_{\alpha}\left(f^{m_{k+r}^{\prime}}(x), q\right) \text {. }
$$

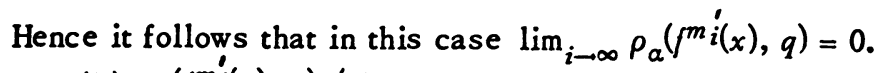

(B) $\rho_{a}\left(f^{m^{\prime}}(x), q\right) \neq 0$ for each $i=1,2, \cdots$.

Then by the first part of condition (ii) of the theorem

$$
\rho_{a}\left(f^{m_{i}^{\prime}}(x), q\right)<\rho_{a}\left(f^{m_{i}^{\prime}-1}(x), q\right)<\cdots<\rho_{a}\left(f^{n^{i}}(x), q\right) \text {. }
$$

Hence $\lim _{i \rightarrow \infty} \rho \rho_{a}\left(f^{m^{\prime}}(x), q\right)=0$ in this case either as $\lim _{i \rightarrow \infty} \rho_{a}\left(f^{n} i(x), q\right)=0$. Thus we have $\lim _{i \rightarrow \infty} \rho_{a}\left(f_{i}^{\prime}(x), q\right)=0$. Now by the triangle inequality,

$$
\rho_{a}(p, q) \leq \rho_{a}\left(p, f^{m_{i}^{\prime}}(x)\right)+\rho_{a}\left(f^{m_{i}^{\prime}}(x), q\right) \text { for } i=1,2, \cdots
$$

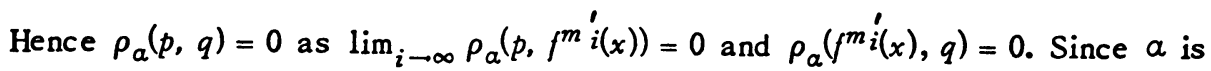
arbitrary, $\rho_{\alpha}(p, q)=0$ for each $\alpha \in I$. Since $X$ is $\tau_{b}$-Hausdorff, this implies that $p=q$.

(d) Finally we prove that if $L(x)$ consists of just one point, then $r_{b}-\lim f^{m}(x)$ exists. Let $\{y\}=L(x)$. Then there is a subnet $\left\{f^{n} j(x)\right\}_{j \in J}$ of the net $\left\{f^{m}(x)\right\}_{m=1}^{\infty}$ such that $\tau_{b}-\lim f^{n} j(x)=y$. Now by Lemma $2.2, f^{n} j(x) \rightarrow y$ in the $\rho_{a}$-topology of $X$ for each $a \in I$. Let $a \in I$ be arbitrarily chosen. Let $\epsilon>0$. Then since $f^{n} j(x)$ $\rightarrow y$ in the $\rho_{a}$ topology of $X$, there is a $s \in J$ such that $\rho_{a}\left(f^{n} j(x), y\right)<\epsilon$ for all $j>s$ and hence for all $n_{j} \geq n_{s}$ where $>$ is the relation in $J$. We now show that for all positive integers $m \geq n_{s}, \rho_{a}\left(f^{m}(x), y\right)<\epsilon$. We have at most two cases:

Case 1. $0=\rho_{a}\left(f^{n_{s}}(x), y\right)<\epsilon$.

Case 2. $0<\rho_{a}\left(f^{n_{s}}(x), y\right)<\epsilon$.

In Case 1, $0=\rho_{a}\left(f^{n_{s}}(x), y\right)=\rho_{a}\left(f^{n_{s}+1}(x), y\right)=\cdots=\rho_{a}\left(f^{m}(x), y\right)<\epsilon$.

In Case 2, $\rho_{a}\left(f^{m}(x), y\right)<\rho_{a}\left(f^{m-1}(x), y\right)<\cdots<\rho_{a}\left(f^{n} s(x), y\right)<\epsilon$.

Thus, in all cases, $\rho_{a}\left(f^{m}(x), y\right)<\epsilon$ whenever $m \geq n_{s}$. Hence $f^{m}(x) \rightarrow y$ in the $\rho_{a}$-topology of $X$. Now since $a$ is arbitrary, $f^{m}(x) \rightarrow y$ in the $\rho_{a}$-topology of $X$ for 
each $a \in I$. Hence by Lemma 2.2, $\tau_{b}-\lim -f^{m}(x)=y$.

Remark 4. The Remark 10 in [2] concerning the work of Edelstein [8, Theorem 1 and 3.2] applies equally here.

Corollary 2.1. Suppose in addition to the bypotheses of Theorem 2.3 that for each $x \in X, L(x) \neq \varnothing$, then for each $x \in X,\left\{f^{n}(x)\right\}_{n=1}^{\infty}$ converges in $\tau_{b}$-topology to a fixed point of $f$.

Proof. This follows immediately from the above Theorem 2.3.

Remark 5. If we assume in the above Theorem 2.3 that $f(X)$ is compact, then this will insure the additional condition assumed in the above corollary, i.e., for each $x \in X, L(x) \neq \varnothing$.

The next theorem is patterned after the Theorem 3.1 of [2].

Theorem 2.4. Let $(X, b)$ be a Hausdorff uniform space and let $\left[\rho_{\alpha}: a \in I\right]=$ $A^{*}(b)$. Let $f: X \rightarrow X$ be $r_{b}$-continuous. Also let

(i) $F(f)$ be nonempty;

(ii) for each $x \in X$ with $x \notin F(f)$ and each $p \in F(f)$, we bave for each $a \in I$, $\rho_{a}(f(x), p) \leq \rho_{a}(x, p)$;

(iii) $f$ be asymptotically regular on $X$.

Then for each $x \in X$, either $\left\{f^{m}(x)\right\}_{n=1}^{\infty}$ contains no $r_{b}$-convergent subnet, or $r_{b}-\lim f^{m}(x)$ exists and belongs to $F(f)$.

Proof. The proof that $L(x) \subset F(f)$ is exactly the same as given in the part (i) of the proof of Theorem 2.1. The proof that $L(x)$ contains at most one point can be obtained from the part (c) of the proof of the above Theorem 2.3 by ignoring the case $A$ and replacing all the strict inequality signs appearing in case $B$ by $\leq$ signs. The rest of the proof of this theorem can be obtained from part (d) of the Theorem 2.3 by ignoring Case 1 and replacing all the strict inequality signs by $\leq$ in the proof of Case 2 .

Corollary 2.2. Let $(X, b)$ be a Hausdorff uniform space and let $\left[\rho_{a}: \alpha \in I\right]=$ $A^{*}(b)$. Let $f$ be an asymptotically regular $\tau_{b}$-continuous mapping of a subset $Y \subset X$ into $Y$. Also suppose that

(i) $f(Y)$ is $\tau_{b} \cdot$ compact;

(ii) for each $x \in Y$ with $x \notin F(f)$, we bave for each $a \in I, \rho_{a}(f(x), p) \leq \rho_{a}(x, p)$.

Then for each $x \in Y$, the sequence $\left\{f^{m}(x)\right\}_{m=1}^{\infty}$ converges in the $\tau_{b}$-topology to a fixed point of $f$.

Proof. Since $f$ is asymptotically regular on $Y$ and $f(Y)$ is compact, we have by Theorem 2.1 that $L(x) \neq \varnothing$ for each $x \in Y$ and $F(f) \neq \varnothing$. Hence the corollary follows from the above Theorem 2.4. 
3. An extended remark. Since our results will concern only with locally convex linear Hausdorff topological spaces, $(E, \tau)$ will denote a locally convex linear Hausdorff topological space throughout the rest of the paper.

It is well known that given $(E, \tau)$, there always exists a family $\left[p_{a}: \alpha \in I\right]$ of seminorms on $E$ which generates the topology $\tau$ in $E$. More specifically, there always exists a family $\left[p_{\alpha}: \alpha \in I\right]$ of seminorms on $E$ such that the family of scalar multiple $r U, r>0$, of finite intersections $U=\bigcap_{k=1}^{n} U_{a_{k}}$, where $U_{a_{k}}=$ $\left\{x: p_{a_{k}}(x) \leq 1\right\}$, forms a neighbourhood base at 0 for the topology $r$ (see $[0$, p. 48], or $[10$, p. 203]).

In the sequel this zero neighbourhood base will be denoted by $\mathfrak{B}$.

Now for each $a \in I$, the function $\rho_{a}: E \times E \rightarrow R$ (the real line) defined by $\rho_{\alpha}(x, y)=p_{\alpha}(x-y)$ for each pair $(x, y) \in E \times E$ is a pseudometric on $E$. Thus by what we have noted in the beginning of $\oint_{1}$ the family $\left[\rho_{\alpha}: \alpha \in I\right]$ of pseudometrics on $E$ (obtained from the family $\left[p_{a}: \alpha \in I\right]$ as above) determines a unique uniformity $b$ on $E$ such that $A^{*}(b)=\left[\rho_{a}: a \in I\right]$. It is well known that $\tau_{b}=\tau$ (e.g. see [9, p. 16]).

Now it is straightforward to see that the following definitions $\left(i^{\prime}\right),\left(i i^{\prime}\right)$ and (iii') are equivalent respectively to definitions (i), (ii) and (iii) given in $\S 1$.

Let $\mathcal{U}$ be the family of zero neighbourhoods in $E$.

Then we have seen that $\mathfrak{B}$ defined above is a base for $\mathcal{U}$. Let $f$ maps $X \subset$ $E$ into $X$, then

(i') $f$ is said to be nonexpansion on $X$ if $x-y \in U$ implies $f(x)-f(y) \in U$ for each $U \in \mathbb{B}$ and $(x, y) \in X \times X$.

(ii') $f$ is said to be a contraction on $X$ if for each $U \in \Re$, there is a real number $r_{U}, 0<r_{U}<1$, such that $x-y \in U$ implies $f(x)-f(y) \in r_{U} U$ for each $U \in \mathfrak{B}$ and $(x, y) \in X \times X$.

(iii') $f$ is asymptotically regular on $X$ if for each $x \in X$ and $U \in \mathcal{U}$, there is a positive integer $n_{0}$ such that $f^{n}(x)-f^{n+1}(x) \in U$ for $n \geq n_{0}$.

[For (i) $\Leftrightarrow\left(\right.$ (i') $^{\prime}$ and (iii) $\Leftrightarrow$ (iii') see Remark 2 and [1]. We prove that (ii') $\Leftrightarrow$ (ii). To prove this we first show that $f$ is a contraction with respect to $A^{*}(b)$. Let $\rho_{a} \in A(b)$ and $(x, y) \in X \times X$. Also let $\rho_{a}(x, y)=r$. Then $x-y \in r U_{a}=U \in \mathbb{B}$ where $U_{a}=\left\{x: p_{a}(x) \leq 1\right\}$. Hence by (ii') there is a real number $r_{U}, 0<r_{U}<1$, such that $f(x)-f(y) \in r_{U} U$. This implies that $\rho_{a}(f(x), f(y)) \leq r_{U} \rho_{a}(x, y)$. Clearly $r_{U}$ depends on $\alpha$. Hence we can write $r_{U}=r(\alpha)$.

Similarly we prove that (ii) $\Rightarrow$ (ii'). This can be done as follows. Let $U \in \mathfrak{B}$. Then $U=r \bigcap_{k=1}^{n} U_{a_{k}}, r>0$ and $U_{a_{k}}=\left\{x: p_{a_{k}}(x) \leq 1\right\}$. Let $\rho_{a_{k}}$ be the corresponding pseudometrics. Choose $r_{U}=\max \left(r\left(\alpha_{k}\right): k=1,2, \cdots, n\right)$ where $\alpha_{k}$ 's are obtained from the definitions (ii).]

Since now we will be concerned with only the locally convex topology $r$ in $E$, unless otherwise stated, all topological concepts such as continuity, conver- 
gence, closedness, etc. will hereafter be meant with respect to the topology $\tau$ in E.

A subset $X$ of $E$ is called starshaped if there is a point $p \in X$ such that for each each $x \in X$ and real $t$ with $0<t<1, t x+(1-t) p \in X . p$ is called the star centre of $X$. Every convex subset of $E$ is thus starshaped.

The following result which we write as a lemma is due to Taylor [1]. However our concept of a contraction mapping and The orem 1.1 provides the following simpler proof.

Lemma 3.1. Let $X$ be a complete bounded starshaped subset of $E$ and letf be nonexpansion on $X$. Then 0 lies in the closure of $(I-f) X$, i.e., in $\overline{(I-f) X}$, where $I$ is the identity map on $X$.

Proof. For each $t, 0<t<1$, we define $f_{t}(x)=t f(x)+(1-t) p, x \in X, p$ being the star centre of $X, f_{t}$ is a self mapping on $X$ as $X$ is starshaped. Let $U \in \mathfrak{B}$ be arbitrary. Let $x-y \in U$. Then $f_{t}(x)-f_{t}(y)=t(f(x)-f(y)) \in t U$ as $f$ is nonexpansion on $X$. Thus $f_{t}$ is a contraction on $X$. Now since $X$ is complete and by our Theorem $1.1 f_{t}$ has a unique fixed point $x_{t}$, say, in $X$. Now

$$
\begin{aligned}
(I-f)\left(x_{t}\right) & =x_{t}-f\left(x_{t}\right) \\
& =x_{t}-\left(f_{t}\left(x_{t}\right)-(1-t) p\right) / t, \text { from the definition of } f_{t}, \\
& =(1-1 / t)\left(x_{t}-p\right) \rightarrow 0 \text { as } t \rightarrow 1 \text {, because } X \text { is bounded. }
\end{aligned}
$$

Remark 6. Note that in the proof of the above lemma we have not used the fact that $X$ is connected.

Let us express the condition (ii) of Theorem 2.4 and condition (ii) of Theorem 2.3 by saying respectively that $f$ is nonexpansion on $X$ with respect to $F(f)$ and $f$ is contractive (in the terminology of [8]) on $X$ with respect to $F(f)$. Thus in $(E, \tau) f$ is nonexpansion on $X \subset E$ with respect to $F(f)$ if $x-p \in U$ implies $f(x)-p \in U$ for each $U \in \mathfrak{B}$ and $x \in X$ with $x \notin F(f)$ and $p \in F(f)$. $f$ is contractive on $X$ with respect to $F(f)$ if the condition (ii) of Theorem 2.3 holds where $\rho_{a}$ 's are obtained from the corresponding $p_{a}$ 's.

Theorem 3.1. Let $f$ be a nonexpansion on a bounded complete starshaped subset $X$ of $E$. Also let $(I-f) X$ be closed. Then $f$ bas a fixed point.

Proof. By Lemma 3.1, $0 \in \overline{(I-f) X}=(I-f) X$. Hence there is a point $p \in X$ such that $(I-f)(p)=0$, i.e. $f(p)=p$.

Corollary 3.1. Let $f$ be a nonexpansion on a compact starshaped subset $X$ of $E$. Also suppose that $f$ is contractive on $X$ with respect to $F(f)$. Then for each $x \in X$, the sequence $\left\{f^{m}(x)\right\}_{m=1}^{\infty}$ converges to a fixed point of $f$. 
Proof. By continuity of $(I-f),(I-f) X$ is compact and hence closed. Now by Theorem $3.1 F(f)$ is nonempty. Also since $f(X)$ is compact, $L(x) \neq 0$ for each $x \in \mathrm{X}$. Hence the corollary follows from Theorem 2.3.

Theorem 3.2. Let $f$ be a continuous asymptotically regular mapping on a closed bounded subset $X$ of $E$. Also suppose that $(I-f)$ maps closed and bounded subsets of $X$ into closed subset of $E$. Then for eacb $x \in X, L(x)$ is nonempty and a closed subset of $F(f)$. If in addition $f$ is nonexpansion on $X$ with respect to $F(f)$, then for each $x \in X$, the sequence $\left\{f^{m}(x)\right\}_{m=1}^{\infty}$ converges to a fixed point of $f$.

Proof. The proof is identical to the proof of Theorem 3.3 in [1] because the nonexpansion of $f$ with respect to $F(f)$ is only used there. Alternatively, we prove in the same way as in Theorem 3.3 of [1] that $F(f) \neq \varnothing$ and $L(x) \neq \varnothing$ for each $x \in X$ and then we refer to our Theorem 2.4 .

Remark 7. Clearly this theorem includes the Theorem 3.3 of [1] which includes the Theorem 6 of Browder and Petryshyn [11]. Also we note that the present theorems weaken the conditions of Theorem 3.3 of [1] in exactly the same way as the Theorem 3.4 of Diaz and Metcalf [2] does to the conditions of Theorem 6 of [11].

Acknowledgment. Professor Rudolf Vyborny drew my attention to the interesting work of Diaz and Metcalf [2] and encouraged me to extend the ir work to uniform spaces. I wish to thank him not only for this but also for all the pains he took in reading the first version of the manuscript of this work.

\section{BIBLIOGRAPHY}

1. W. W. Taylor, Fixed-point theorems for nonexpansive mappings in linear topological spaces, J. Math. Anal. Appl. 40 (1972), 164-173.

2. J. B. Diaz and F. T. Metcalf, On the set of subsequential limit points of successive approximations, Trans. Amer. Math. Soc. 135 (1969), 459-485.

3. F. Tricomi, Un teorema sulla convergenza delle successoni formate delle successive iterate di una funzione di una variabile reale, Giorn. Mat. Battaglini 54 (1916), 1-9.

4. D. G. Defigueiredo, Topics in nonlinear functional analysis, Lecture series No. 48, University of Maryland Press, College Park, Md. 1967.

5. J. L. Kelley, General topology, Van Nostrand, Princeton, N. J., 1955. MR 16, 1136.

6. W. J. Thron, Topological structures, Holt, Rinehart and Winston, New York, 1966. MR 34 \#778.

7. H. Schubert, Topologie. Eine Einführung, Teubner, Stuttgart, 1964; English transl., Allyn and Bacon, Boston, Mass., 1968. MR 30 \#51; 37 \#2160.

8. M. Edelstein, On fixed and periodic points under contractive mappings, J. London Math. Soc. 37 (1962), 74-79. MR 24 \#A2936.

9. H . H. Schaefer, Topological vector spaces, Macmillan, New York, 1966. MR 33 $\# 1689$. 
10. G. Köthe, Topologische linear Räume. I, Die Grundlehren der math. Wissenschaften, Band 107, Springer-Verlag, Berlin, 1960; English transl., Die Grundlehren der math. Wissenschaften, Band 159, Springer-Verlag, New York, 1969. MR 24 \#A411; 40 \#1750.

11. F. F. Browder and W. V. Petryshyn, The solution by iteration of nonlinear functional equations in Banach spaces, Bull. Amer. Math. Soc. 72 (1966), 571-575. MR 32 \#8155b.

DEPARTMENT OF MATHEMATICS, UNIVERSITY OF QUEENSLAND, ST. LUCIA, BRISBANE, QUEENSLAND, 4067, AUSTRALIA 